\title{
TRANSFERS OF CARE AROUND MEDICINE
}

\author{
C Learoyd $\mathrm{MSc}^{1,2}$ \& B Crotty PhD, CPFA \\ ${ }^{1} \mathrm{HCPC}$ registered Physiotherapist \\ ${ }^{2}$ Project Manager for the West of England AHSN \\ ${ }^{3}$ Corporate Development Manager for the West of England AHSN
}

There are 15 Academic Health Science Networks (AHSNs) across England, established by NHS England in 2013. They were formed in order to spread innovation at pace and scale, thereby improving health and generating economic growth. Each AHSN works across a distinct geography serving a different population in each region. Patient Safety is one of the key priorities of the AHSNs. Fundamental aspects of Patient Safety include ensuring that medication is used to its maximum benefit, and reducing medicine associated risks.

One project covering both patient safety issues and optimising medicine use is Transfers of Care around Medicine (TCAM). When a patient moves from one care setting to another, this is known as a transfer of care. When transfers of care occur there is an increased risk of adverse effects. $30-70 \%{ }^{1}$ of patients experience unintentional changes to their treatment or an error is made because of a miscommunication. The TCAM project in the West of England AHSN has evolved, in part, to support initiatives to improve discharges and potentially free up capacity in response to Covid 19. The focus has been to improve communications to care home pharmacy teams in response to the NHS England 'call to action'. TCAM into care homes pharmacy teams is a joint project between hospitals, primary care teams and commissioners to ensure the impacts of changes, and any adverse effects, are minimised following the discharge of a patient from hospital to a care home. The October 2019
"Healthcare Finance" article "On the Way Down"2 highlighted the work of South Sefton/Southport and Formby CCGs' Medicines Management Team. The team were working to “review, optimise and reconcile patients' medicines after hospital discharge". This is the key component of the TCAM project in the West of England.

There have been many studies that highlight the impacts of medication error following a patient's discharge from hospital. A recent study (Elliott et al) ${ }^{3}$ estimated that there were 441,000 "severe" medication errors in care homes in England in 2015/16. One key risk factor noted was the increased chance of errors occurring after discharge when the patient is supplied with a multi-compartmental compliance aid and is placed or returns to a care home environment. The TCAM system involves identifying patients in hospital who need additional support with their medicines, most commonly for people using multi-compartmental compliance aids. These aids are designed to support patients in taking medications, by ensuring compliance/ adherence to the prescribed dosage. At the point of discharge patients are referred, via the PharmOutcomes ${ }^{4}$ secure digital platform. The community pharmacist receives a direct notification of the discharge prescription for that patient. This triggers a review and reconciliation of the patient's medication and alerts the pharmacist to advise the patient of any changes. Communicating at an earlier stage 
with the community pharmacist has significant potential to reduce waste, especially with verifying the contents of a multi-compartmental compliance aid that could have been assembled without the community pharmacist being informed of the most recent changes. TCAM will therefore be extended to provide advanced information via a regionally nominated care home pharmacy team, to allow additional rigour and safety in supporting the administration of the medication to the resident. The flow of information is shown below.

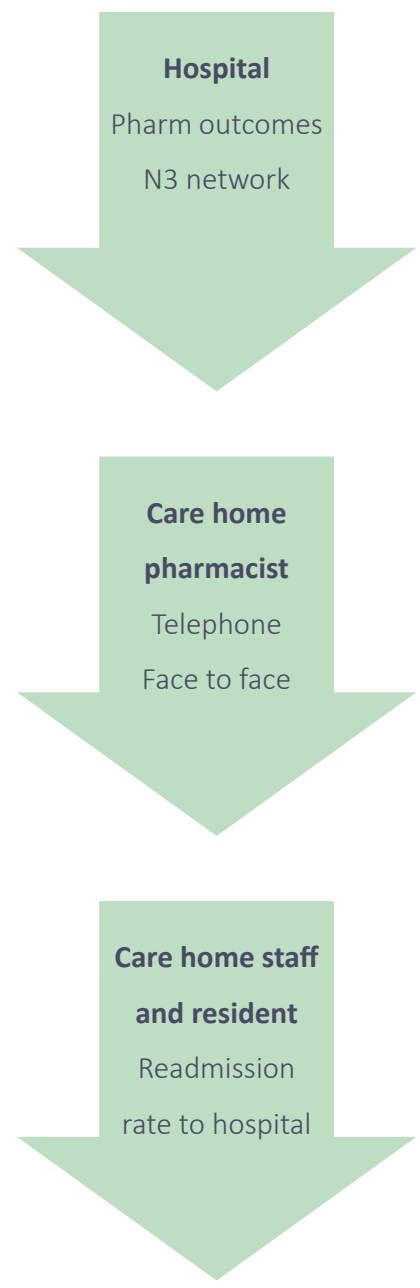

The West of England AHSN is working with staff from all backgrounds including pharmacists liaising with care home staff, clinicians, primary care staff and accountants to develop and implement the PharmOutcomes management system. This will automatically capture the relevant information and disseminate this to the required healthcare professional. A one year licence for the software costs $£ 4-5,000$ p.a. (plus VAT), depending on the number of sites within the organisation. A communications link to any number of care homes pharmacy teams within the patch would cost $£ 2,000$ p.a. There are very few other on-going operational costs and, as part of this initiative, the West of England AHSN will fund the first year's PharmOutcomes subscription to effectively "pump-prime" the project.

The key benefits for the care home TCAM project will be to minimise the chances of prescribing and consequent administration errors. These errors can result in patients being readmitted to hospital for, at best, observation or, more seriously, treatment requiring medical intervention and an in-patient stay. The cost of a readmission following an "avoidable adverse drug event" can vary significantly; Sabir et al's ${ }^{5}$ survey noted $37 \%$ of older patients experience medication related harm within eight weeks of discharge. This was estimated to cost the NHS $£ 396 m$ p.a. Given the increasing numbers of discharges to care homes TCAM can go some way to addressing this issue.

The costs reported in studies associated with the avoidance of errors are mainly average costs and utilise published calculations such as HRG (healthcare resource group) reference costs ${ }^{6}$. Morris et $\mathrm{al}^{7}$, noted that, in estimating costs from both a financial and economic perspective: "Average cost data obscure potentially important issues with respect to the technical efficiency of providers". Given a significant proportion of primary and secondary care NHS costs are fixed in the short to medium term there are few realisable savings that will be immediately identifiable within e.g. an individual Trust budget. These savings would include any variable costs in the ED/assessment unit (medication, oxygen, consumables) and avoiding any variable accommodation costs if an inpatient stay is required following readmission. There would be additional cost avoidance in lower levels of ambulance conveyancing and for GP consultations/contacts. On a wider aspect medication errors can also impact on patient mobility and susceptibility to infections; leading to a higher incidence of falls or additional treatments to clear infections.

It is therefore likely the immediately identifiable variable costs of avoiding one readmission are relatively small when undertaking a financial analysis; thereby potentially distorting a traditional "return on investment" analysis. Costs avoided may not be identifiable until at least six months after until the post implementation activity is analysed. What is clear, however, is that, all other things being equal, avoiding a readmission following an adverse drug event will free up hospital capacity and place less strain on hospital staff. These economic, rather than financial, costs are often based on 
averages and used to illustrate opportunity costs. Sabir et al ${ }^{5}$ quote an "excess bed day" as costing $£ 313$ from an economic perspective. Additionally, from a societal perspective, any adverse event places increased emotional strain on the individual's family and will, arguably, have a knock on effect on their productive capacity by e.g. taking time away from work to visit their relative in hospital. Accountants are not directly involved with quantifying these factors however most of them should be able to relate to these societal impacts.

Activity information on readmissions is challenging to quantify. The House of Commons Public Accounts Committee (PAC) reported in May $2018^{8}$ that readmission activity could not be relied upon to be "an indicator of failures in care".

The PAC noted that "It is lamentable that nearly 1.5 million people could have avoided emergency admissions in 2016-17 if hospitals, GPs, community services and social care had worked together more effectively". NHS Digital ${ }^{9}$ have been developing the reporting mechanisms so it is safe to say that more granular information should be available going forward to support analysis relating to this initiative.

In the West of England this project has the direct input from Clinical Pharmacy Directors, Local Pharmacy Committee executives and CCG health professionals, working collaboratively with the Royal United Hospitals, Bath NHSFT, North Bristol Trust and University Hospitals Bristol/Weston NHSFT. These Trusts, along with Great Western Hospital NHSFT and Gloucestershire Hospitals NHSFT, are involved in setting up the care home element of TCAM and we will expect to see some measurable benefits accruing later this financial year.

We will additionally work with partners in the National AHSN network and other research organisations to develop and estimate the financial and economic benefits from adopting the TCAM initiative. Minimising emergency admissions/ readmissions due to adverse drug events associated with a patient's transfer of care can only have positive impacts whatever form of analysis is undertaken to support the initiative. Accountants, health economists and clinicians can all therefore jointly pursue the common goal of generating additional Covid 19 capacity, avoiding unnecessary costs and, at the same time, enhancing patient safety for patients.

\section{DECLARATION OF INTERESTS:}

Neither author has any conflict of interest nor any financial/ commercial interests or individual commercial relationships with Pinnacle Health Partnership LLP or any of its associated/ related companies.

\section{ACKNOWLEDGEMENT:}

The authors wish to express their thanks to Elin Crotty, School of Literatures, Languages \& Cultures, University of Edinburgh for final proof reading.

\section{REFERENCES:}

1. West of England AHSN. Transfers of Care Around Medicine. [WWW] https://www.weahsn.net/ourwork/transforming-services-and-systems/medicinesoptimisation/transfers-of-care-around-medicines/ (accessed 16 July 2020)

2. Healthcare Finance, On the Way Down. HFMA October 2019

3. Elliott RA, Camacho E, Jankovic D, Sculpher MJ, Faria R. Economic analysis of the prevalence and clinical and economic burden of medication error in England. BMJ Qual Saf 2020;0:1-10. https://doi:10.1136/ bmjqs-2019-010206

4. PharmOutcomes system. Provided by Pinnacle Health Partnership LLP. [WWW] See https://pharmoutcomes.org (accessed 16 July 2020)

5. Sabir FR, Tomlinson J, Strickland-Hodge B, Smith H. Evaluating the Connect with Pharmacy web-based intervention to reduce hospital readmission for older people. International Journal of Clinical Pharmacy. https://doi.org/10.1007/s11096-019-00887-3

6. NHS Improvement. National Cost Collection for the NHS. [WWW] https://improvement.nhs.uk/resources/nationalcost-collection/ (accessed 16 July 2020) 
7. Morris S, Devlin N, Parkin D (2012), Economic Analysis in Health Care. Wiley 2012

8. House of Commons Committee of Public Accounts.

Reducing Emergency Admissions-Forty Fourth Report of Session 2017-19 [WWW] https://publications.parliament. uk/pa/cm201719/cmselect/cmpubacc/795/795.pdf (accessed 16 July 2020)

9. NHS Digital. Compendium-Emergency Readmission to Hospitals within 30 Days of Discharge. https://digital. nhs.uk/data-and-information/publications/clinicalindicators/compendium-of-population-health-indicators/ compendium-hospital-care/current/emergencyreadmissions-to-hospital-within-30-days-of-discharge (accessed 16 July 2020) 